\title{
CIENCIAMATRIA
}

Revista Interdisciplinaria de Humanidades, Educación, Ciencia y Tecnología

Año VIII. Vol. VIII. Nro 1. Edición Especial. 2022

Hecho el depósito de ley: pp201602FA4721

ISSN-L: 2542-3029; ISSN: 2610-802X

Universidad Nacional Experimental Francisco de Miranda (UNEFM). Santa Ana de Coro. Venezuela

Ángel Alberto Dionisio-Cuadrado

DOI $10.35381 / \mathrm{cm} . \mathrm{v} 8 \mathrm{i} 14.658$

\section{Relación del sistema de gestión de riesgos con índice de accidentabilidad en} empresa de hidrocarburos

Relationship of the risk management system with accident rate $\mathbf{i}$ a hydrocarbon company

\author{
Ángel Alberto Dionisio-Cuadrado \\ adionisio77@gmail.com \\ Investigador Independiente \\ Perú
}

https://orcid.org/0000-0002-5554-3948

Recibido: 15 de septiembre 2021

Revisado: 10 de noviembre 2021

Aprobado: 15 de diciembre 2021

Publicado: 01 de enero de 2022 


\author{
CIENCIAMATRIA \\ Revista Interdisciplinaria de Humanidades, Educación, Ciencia y Tecnología \\ Año VIII. Vol. VIII. Nro 1. Edición Especial. 2022 \\ Hecho el depósito de ley: pp201602FA4721 \\ ISSN-L: 2542-3029; ISSN: 2610-802X \\ Universidad Nacional Experimental Francisco de Miranda (UNEFM). Santa Ana de Coro. Venezuela
}

Ángel Alberto Dionisio-Cuadrado

\title{
RESUMEN
}

El presente artículo tiene como objetivo analizar la aprobación de la aplicación del sistema de gestión de riesgo como estrategia para mejorar la gestión en la seguridad y la salud ocupacional, que se concretó con la reducción de la accidentabilidad como se aprecia en los resultados logrados lo que se estima y observa en la aplicación en el presente estudio, mediante el análisis directo con la finalidad de considerar como la inserción del control de la gestión del riesgo significó un aporte notable para mejorar la gestión de los planes de seguridad y salud: prevención, Corrección, Respuesta, de tal forma que se ha obtenido un resultado que permitió la validación de una estrategia que propicie la reducción de la incidencia de los accidentes, otorgando con un componente que siempre se tiene que a considerar hacia adelante.

Descriptores: Medida de seguridad; prevención de accidentes; dispositivo de seguridad. (Tesauro UNESCO).

\begin{abstract}
This article aims to analyze the approval of the implementation of the risk management system as a strategy to improve management in occupational safety and health, which materialized with the reduction of the accident rate as seen in the results achieved what is estimated and observed in the application in this study, through direct analysis in order to consider how the insertion of risk management control meant a significant contribution to improve the management of safety and health plans: Prevention, Correction, Response, in such a way that a result has been obtained that allowed the validation of a strategy that propitiates the reduction of the incidence of accidents, granting with a component that always has to to be considered forward.
\end{abstract}

Descriptors: Safety measures; accident prevention; safety devices. (UNESCO Thesaurus). 


\section{CIENCIAMATRIA \\ Revista Interdisciplinaria de Humanidades, Educación, Ciencia y Tecnología \\ Año VIII. Vol. VIII. Nro 1. Edición Especial. 2022 \\ Hecho el depósito de ley: pp201602FA4721 \\ ISSN-L: 2542-3029; ISSN: 2610-802X \\ Universidad Nacional Experimental Francisco de Miranda (UNEFM). Santa Ana de Coro. Venezuela}

Ángel Alberto Dionisio-Cuadrado

\section{INTRODUCCIÓN}

El presente artículo basado en un estudio analiza la relación del sistema de gestión de riesgos (SGR) y el índice de accidentabilidad (IA), tomando como referente los planteamientos y acciones del ISO 45001:2018 que postula a resolver la problemática en el incremento de los accidentes y los índices que al respecto se tiene y son una constante preocupación (Miño-Cascante et al 2020), este estudio entonces muestra el proceso que llevo al surgimiento de un cuestionamiento relacionado con identificar la influencia favorable del SGR en el IA.

El desarrollo de este estudio ha requerido que se considere los objetivos del estudio que tiene que ver con determinar la influencia significativa del SGR en la reducción del IA de la empresa del sub sector hidrocarburos EBH Ingenieros S.A.C. para ello además está explicando las condiciones del sistema de gestión de riesgos implementada en la empresa del sub sector hidrocarburos EBH Ingenieros S.A.C. y estableciendo el índice de accidentabilidad de los trabajadores de la Empresa del Sub Sector Hidrocarburos EBH Ingenieros S.A.C.

Antes y después de la implementación del Sistema de riesgo, eso implica explicar que se evalúa antes y después de implementar el SGR, lo propuesto da cuenta de una investigación de tipo aplicada que busca explicar y comprender esa relación fundamental entre la implementación de un SGR y de reducir los IA. Demostrando y comprobando que la hipótesis planteada era válida.

La información para esta investigación provino de fuentes primarias en la aplicación de información recogida en campo, pero también de carácter secundario, basado en reportes existentes y artículos de revistas especializadas y libros que analizan la relación Sistema - Accidentabilidad, todo ello para poder reflexionar y determinar que es necesario implementarlos para lograr disminuir los eventos adversos, favoreciendo la situación de la seguridad y tranquilidad de las personas. Con respecto al estudio se presentan los cuadros principalmente que demuestran la propuesta y su importancia, 


\section{CIENCIAMATRIA \\ Revista Interdisciplinaria de Humanidades, Educación, Ciencia y Tecnología \\ Año VIII. Vol. VIII. Nro 1. Edición Especial. 2022 \\ Hecho el depósito de ley: pp201602FA4721 \\ ISSN-L: 2542-3029; ISSN: 2610-802X \\ Universidad Nacional Experimental Francisco de Miranda (UNEFM). Santa Ana de Coro. Venezuela}

Ángel Alberto Dionisio-Cuadrado

para luego en resumidas cuentas advertir algunos hechos que tienen que ver con el estudio y se realizaron para lograr la obtención de la información mediante las encuestas, las observaciones, que permitió comprender los hechos.

En este sentido, el proceso de implementar el sistema de gestión de riesgos, explicando su necesidad, de que espacio se inicia, con que perspectiva o visión comprendiendo la prevención y la actitud responsable y comprometida, apreciando el uso de las herramientas sobre los procesos que dan lugar a consolidad la seguridad, mostrando cuales son las perspectivas que puedan ser asumidas y generalizadas en función de los trabajadores en cualquier entidad y/o empresa. (Arbaiza, 2014).

Por lo tanto, generar este tipo de estudios es importante puesto que permitió demostrar toda la importancia que el mismo ha tenido como establecer los procedimientos que permiten aportar tanto en lo teórico y práctico con la implementación de la seguridad y salud en el trabajo, de hecho, esta información es una motivación para mejorar estos conocimientos. Por consiguiente, se cuenta con referencias de las situaciones que se viven, incidentes, lesiones o muertes, como precisa la Organización Internacional del Trabajo:

Cada día mueren personas a causa de accidentes laborales o enfermedades relacionadas con el trabajo - más de 2,78 millones de muertes por año. Además, anualmente ocurren unos 374 millones de lesiones relacionadas con el trabajo no mortales, que resultan en más de 4 días de absentismo laboral. El coste de esta adversidad diaria es enorme y la carga económica de las malas prácticas de seguridad y salud se estima en un 3,94 por ciento del Producto Interior Bruto global de cada año. (OIT, 2021).

En consecuencia, existen patrones o estándares de accidentes y muertes, en lo que se refiere que las caídas a desnivel son la segunda causa mundial de muerte por lesiones sean estas accidentales o no intencionales. Al respecto se tiene lo siguiente: "Se calcula que anualmente mueren en todo el mundo unas 646000 personas debido a caídas, y más de un $80 \%$ de esas muertes se registran en países de bajos y medianos recursos" (PUCP - OSHAS, 2018). Por otro lado, es conveniente promover un proceso formativo y 


\section{CIENCIAMATRIA \\ Revista Interdisciplinaria de Humanidades, Educación, Ciencia y Tecnología \\ Año VIII. Vol. VIII. Nro 1. Edición Especial. 2022 \\ Hecho el depósito de ley: pp201602FA4721 \\ ISSN-L: 2542-3029; ISSN: 2610-802X \\ Universidad Nacional Experimental Francisco de Miranda (UNEFM). Santa Ana de Coro. Venezuela}

Ángel Alberto Dionisio-Cuadrado

de creación de entornos de trabajos más seguros, esto con la intención de superar el alto nivel personas que padecen accidentes laborables por caídas en su lugar de trabajo, por cuanto "el $0.05 \%$ de los accidentes corresponden a caídas a desnivel en estaciones de servicio, esto quiere decir que 3230 personas ..." (OMS, 2018).

El Perú tiene los mismos problemas, los de caídas de altura, de desnivel son siempre consecuencia de accidentes generalmente, "en cuanto accidentes laborales en el Perú, se evidencia estadísticamente, comparando el año 2018 - 2019; analizando la tendencia de los datos existe una correlación positiva alta, entre el tiempo y el número de accidentes" (Diaz-Dumont et al. 2020, p. 327), ante esos hechos se requería determinar la importancia que tiene implementar el sistema de gestión de riesgos para reducir la accidentabilidad de una empresa, en este caso precisando que el problema es en una empresa de hidrocarburos.

\section{MÉTODO}

\section{Tipo de investigación}

El presente estudio es de investigación aplicada, por la implementación o uso del plan del Sistema de Gestión de Riesgos y cómo influye en los índices de Accidentabilidad, por lo que se aplicó un estudio correlacional pudiendo determinar una puntuación alta en una variable también tiene puntuación alta en una segunda variable y si individuos con una baja puntuación en una variable también tienen baja puntuación en la segunda. Estos resultados indican una relación positiva." (Ramírez, 2004, p. 22). Teniendo entonces el carácter analítico de dos variables.

\section{Hipótesis}

Se ha planteado en el presente estudio: "El sistema de riesgos influye significativamente en la reducción del índice de accidentabilidad de la empresa del subsector de hidrocarburos EBH Ingenieros S.A.C." 


\author{
CIENCIAMATRIA \\ Revista Interdisciplinaria de Humanidades, Educación, Ciencia y Tecnología \\ Año VIII. Vol. VIII. Nro 1. Edición Especial. 2022 \\ Hecho el depósito de ley: pp201602FA4721 \\ ISSN-L: 2542-3029; ISSN: 2610-802X \\ Universidad Nacional Experimental Francisco de Miranda (UNEFM). Santa Ana de Coro. Venezuela
}

Ángel Alberto Dionisio-Cuadrado

Entonces se trata de comprender cuál es la problemática en las variadas complicaciones del sistema de gestión de riesgos y su implementación y determinar además del índice de accidentabilidad que mejora es decir se reduce luego de implementar el Sistema de riesgo en la empresa subsector hidrocarburos EBH Ingenieros

\title{
Variables de la investigación
}

La presente investigación cuenta con las siguientes variables:

\section{Variable dependiente: sistema de riesgos}

Este procedimiento que se aplica de manera experimental dentro del protocolo de seguridad y salud es un proceso que permite señalar cuáles son los riesgos, priorizarlos, atenderlos y considerarlos a fin de prevenir, pero además estar preparados, esto no está al margen de lo existente, sino atiende las condiciones en que el proceso se desarrolla para poder reducir el índice de accidentabilidad preferentemente.

El sistema general de riesgos laborales es el conjunto de entidades públicas y privadas, normas y procedimientos, destinados a prevenir, proteger y atender a los trabajadores de los efectos de las enfermedades y los accidentes que pueden ocurrirles con ocasión o como consecuencia del trabajo que desarrollan. (SURA, 2020).

Se trata de determinar qué actividades permiten establecer acciones de promoción y prevención propensos a optimizar las circunstancias de trabajo y salud del personal trabajador, salvaguardando su integridad de todo riesgo laboral individual o colectivo de cualquier índole: físicos, químicos, biológicos, ergonómicos, psicosociales, de saneamiento y de seguridad, este criterio dentro del trabajo entonces es una situación favorable que antes que atender accidentes los previene o cuenta con una respuesta inmediata y efectiva. 


\section{CIENCIAMATRIA}

Revista Interdisciplinaria de Humanidades, Educación, Ciencia y Tecnología

Año VIII. Vol. VIII. Nro 1. Edición Especial. 2022

Hecho el depósito de ley: pp201602FA4721

ISSN-L: 2542-3029; ISSN: 2610-802X

Universidad Nacional Experimental Francisco de Miranda (UNEFM). Santa Ana de Coro. Venezuela

Ángel Alberto Dionisio-Cuadrado

\section{Variable independiente: Índice de accidentabilidad}

Es el registro de la data existente entre la cantidad de trabajadores y los accidentes que se producen que se establece cuál es el nivel recurrente de los mismos.

Un indicador de accidentalidad puede definirse como una magnitud utilizada para medir o comparar los resultados obtenidos en la empresa en relación con los accidentes, los días perdidos por baja, etc. En definitiva, es el resultado cuantitativo de comparar dos variables. (Aragón, 2016).

Tabla 1.

Operacionalización de variables.

\begin{tabular}{|c|c|c|c|c|c|}
\hline It & Variables & $\begin{array}{c}\text { Definición } \\
\text { Operacional }\end{array}$ & Dimensiones & Indicadores & Instrumento \\
\hline \multirow{7}{*}{ 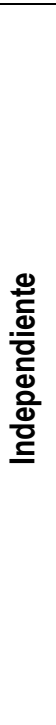 } & \multirow{7}{*}{ 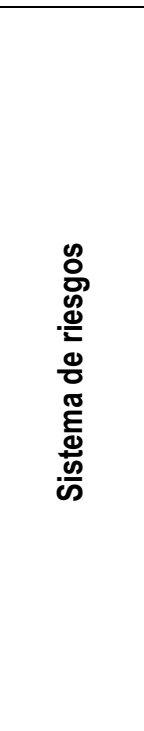 } & \multirow{7}{*}{$\begin{array}{l}\text { Organización } \\
\text { relativamente } \\
\text { duradera de } \\
\text { protocolos y } \\
\text { lineamientos en } \\
\text { torno al control de } \\
\text { los riesgos } \\
\text { existentes en un } \\
\text { entorno laboral } \\
\text { específico }\end{array}$} & \multirow[b]{2}{*}{ Planeamiento } & Organización de & Entrevista al Comité \\
\hline & & & & $\begin{array}{l}\text { equipos. } \\
\text { Determinación de } \\
\text { acciones }\end{array}$ & $\begin{array}{l}\text { Plan de } \\
\text { implementación }\end{array}$ \\
\hline & & & Temporalidad & $\begin{array}{l}\text { Cumplimiento de } \\
\text { acciones en el tiempo } \\
\text { indicado }\end{array}$ & Lista de seguimiento \\
\hline & & & Conocimiento & $\begin{array}{l}\text { Datos e información } \\
\text { que se encuentra } \\
\text { establecida en el } \\
\text { documento. }\end{array}$ & $\begin{array}{l}\text { Encuesta a los } \\
\text { trabajadores } \\
\text { Validación del Plan }\end{array}$ \\
\hline & & & Financiamiento & $\begin{array}{l}\text { Recursos con que } \\
\text { cuenta el Sistema para } \\
\text { su implementación }\end{array}$ & $\begin{array}{l}\text { Informe económico de } \\
\text { inversión }\end{array}$ \\
\hline & & & \multirow{2}{*}{ Viabilidad } & $\begin{array}{l}\text { Estudio de } \\
\text { condiciones favorables }\end{array}$ & $\begin{array}{l}\text { Check list de } \\
\text { obligatorio }\end{array}$ \\
\hline & & & & $\begin{array}{l}\text { o no para su } \\
\text { aplicación }\end{array}$ & $\begin{array}{l}\text { cumplimiento de } \\
\text { criterios }\end{array}$ \\
\hline \multirow{3}{*}{ 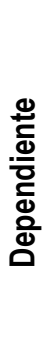 } & \multirow{3}{*}{ 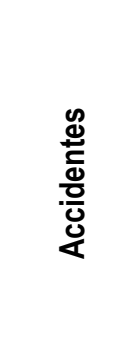 } & \multirow{3}{*}{$\begin{array}{l}\text { Situaciones que se } \\
\text { presentan desde } \\
\text { incidentes hasta } \\
\text { condiciones fatales } \\
\text { que se producen en } \\
\text { un determinado } \\
\text { espacio y dañan de } \\
\text { alguna manera al } \\
\text { trabajador. }\end{array}$} & Índices & $\begin{array}{l}\text { Índices de resultados } \\
\text { de seguridad (índices } \\
\text { de frecuencia, } \\
\text { severidad, } \\
\text { accidentabilidad) }\end{array}$ & $\begin{array}{l}\text { Matriz de identificación } \\
\text { de peligros y } \\
\text { evaluación de riesgos. }\end{array}$ \\
\hline & & & Factores & $\begin{array}{l}\text { Causas probables de } \\
\text { accidentes }\end{array}$ & $\begin{array}{l}\text { Registro de incidentes } \\
\text { y accidentes. }\end{array}$ \\
\hline & & & Consecuencias & $\begin{array}{l}\text { Costos de los } \\
\text { accidentes suscitados. }\end{array}$ & $\begin{array}{l}\text { Informe de los } \\
\text { accidentes suscitados. }\end{array}$ \\
\hline
\end{tabular}




\section{CIENCIAMATRIA \\ Revista Interdisciplinaria de Humanidades, Educación, Ciencia y Tecnología \\ Año VIII. Vol. VIII. Nro 1. Edición Especial. 2022 \\ Hecho el depósito de ley: pp201602FA4721 \\ ISSN-L: 2542-3029; ISSN: 2610-802X \\ Universidad Nacional Experimental Francisco de Miranda (UNEFM). Santa Ana de Coro. Venezuela}

Ángel Alberto Dionisio-Cuadrado

Por consiguiente, determinar la relación es un hecho fundamental de acuerdo con las circunstancias y la situación, al relacionarlo y determinar la gravedad del riesgo se toma conciencia de este, se actúa preventivamente y ello procura que la persona actúe tratando de evitar o prevenir la materialización del riesgo y se evita un accidente. Para poder lograr estos resultados se ha aplicado la operacionalización de las variables para poder desarrollar el estudio de la manera sistemática y técnica:

\section{Unidad de análisis}

La presente investigación define dos unidades de análisis:

A. El sistema de riesgos que garantiza la seguridad de los trabajadores de la que laboran en la empresa EBH Ingenieros SAC.

B. La ocurrencia de accidentes que registra la empresa EBH Ingenieros SAC.

Ambas unidades se relacionaron en el sentido de que a mejoras condiciones del sistema de seguridad, menor el índice de accidentabilidad, entonces se podría determinar cómo la población del estudio de investigación a todas las personas que laboran en empresa EBH Ingenieros SAC, siendo 53 personas, y de ella se desprende 32 personas para realizar el estudio, cuya determinación ha sido sistemática y basado en la estadística: empleando la línea de soldadura de tanques y de armado de tanques, se ha realizado el estudio en los dos turnos rotativos.

\section{Técnicas e instrumentos de recolección de datos}

En la presente investigación se emplearon las técnicas de recolección de datos, en primer orden la observación, siendo aplicada por la gestión de supervisión en la muestra quienes emplearán como instrumento una cartilla de reporte. En esta cartilla, se registrarán todos los actos y condiciones inseguras que identifiquen en las zonas y/o líneas donde se llevará a cabo la investigación. En segundo orden, se aplicó un instrumento tipo cuestionario en escala Likert de varias alternativas de respuestas, mediante la encuesta 


\section{CIENCIAMATRIA}

Revista Interdisciplinaria de Humanidades, Educación, Ciencia y Tecnología

Año VIII. Vol. VIII. Nro 1. Edición Especial. 2022

Hecho el depósito de ley: pp201602FA4721

ISSN-L: 2542-3029; ISSN: 2610-802X

Universidad Nacional Experimental Francisco de Miranda (UNEFM). Santa Ana de Coro. Venezuela

Ángel Alberto Dionisio-Cuadrado

a la muestra poblacional, este fue validado por juicio de expertos y Alfa de Cronbach de 0,88 siendo considerado apto para su aplicación. (Hernández-Sampieri et al. 2014).

\section{Procesamiento estadístico de la información}

La información que se presentó y empleó fue, por las medidas de tendencia central: media aritmética. mediana y moda, empleando, además: cuadros de doble entrada, diagramas de distribución, histogramas y polígonos de frecuencia.

\section{RESULTADOS}

El proceso de análisis se ha determinado procesando en primer lugar el análisis de valorar el sistema de gestión de riesgos, mediante una tabla, apreciando cada uno de los componentes:

\section{Tabla 2.}

Componentes y aspectos del sistema de gestión de riesgos.

\begin{tabular}{ll}
\hline \multicolumn{1}{c}{ Componentes } & \multicolumn{1}{c}{ Aspectos } \\
\hline Compromiso e Involucramiento & Principios \\
Política de Seguridad y Salud en el Trabajo & Política \\
& Dirección \\
& Liderazgo \\
& Organización \\
& Competencia \\
Planeamiento y Aplicación & Diagnóstico \\
Implementación y Operación & Planeamiento para la identificación de peligros, evaluación y \\
& control de riesgos \\
& Objetivos \\
& Plan y programa de Seguridad y Salud en el trabajo \\
& Estructura y responsabilidades \\
& Capacitación \\
Evaluación normativa & Medidas de prevención \\
Verificación & Preparación y respuesta ante emergencias \\
& Requisitos legales y de otro tipo \\
& Accidentes, incidentes peligrosos e incidentes, No \\
& conformidad, Acción correctiva y preventiva \\
& Control de las operaciones \\
& Control de operaciones \\
& Documentos \\
& Control de la documentación y los datos \\
\hline
\end{tabular}




\section{CIENCIAMATRIA}

Revista Interdisciplinaria de Humanidades, Educación, Ciencia y Tecnología

Año VIII. Vol. VIII. Nro 1. Edición Especial. 2022

Hecho el depósito de ley: pp201602FA4721

ISSN-L: 2542-3029; ISSN: 2610-802X

Universidad Nacional Experimental Francisco de Miranda (UNEFM). Santa Ana de Coro. Venezuela

Ángel Alberto Dionisio-Cuadrado

\begin{tabular}{cl}
\hline \multicolumn{1}{c}{ Componentes } & \multicolumn{1}{c}{ Aspectos } \\
\hline & Gestión de los registros. \\
Revisión por la dirección & Gestión de la mejora continua \\
\hline
\end{tabular}

Elaboración: El autor.

Con la ayuda de esa tabla se desarrolla estos resultados que evidencian lo logrado en la implementación del Sistema (Figura 1 y Figura 2).

\section{Tabla 3.}

Línea Base: cumplimientos - incumplimientos del sistema de gestión de riesgos.

\begin{tabular}{ccc}
\hline Respuesta & $\mathbf{f}$ & $\mathbf{p}$ \\
\hline Sí & 15 & 19.74 \\
No & 59 & 77.63 \\
No aplica & 2 & 2.63 \\
$\Sigma$ & 76 & 100.00 \\
\hline
\end{tabular}

Fuente: Tabla 2 - Matriz. Elaborado: febrero 2019.

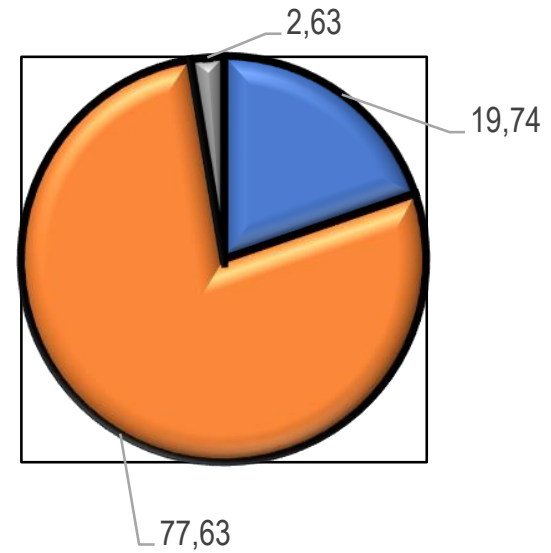

Figura 1. Cumplimiento - incumplimiento del sistema de gestión de riesgos. 
CIENCIAMATRIA

Revista Interdisciplinaria de Humanidades, Educación, Ciencia y Tecnología

Año VIII. Vol. VIII. Nro 1. Edición Especial. 2022

Hecho el depósito de ley: pp201602FA4721

ISSN-L: 2542-3029; ISSN: 2610-802X

Universidad Nacional Experimental Francisco de Miranda (UNEFM). Santa Ana de Coro. Venezuela

Ángel Alberto Dionisio-Cuadrado

\section{Tabla 3.}

Ponderación.

\begin{tabular}{lc}
\hline \multicolumn{2}{c}{ Rangos de puntaje (\%) } \\
\hline Excelente & $91-$ \\
Bueno & 100 \\
Bajo & $81-90$ \\
Inaceptable & $61-80$ \\
\hline
\end{tabular}

Como se aprecia el resultado de cumplimientos está en el rango de $19,74 \%$ que significa Bajo, por tanto es inaceptable, es decir que el sistema de gestión de riesgos en las condiciones que se encuentra no satisface o cumple las condiciones básicas de un Sistema de prevención, por lo que prima su mejoramiento o condiciones básicas de seguridad que mejoren esta situación, ya que el incumplimiento es de $77,63 \%$ de acciones o lineamientos que no se cumplen y 2 que no aplican por la naturaleza de la gestión, de esta manera el alcance es muy bajo, y que se cumplan las acciones de previsión o para evitar accidentes es demasiado bajas.

\section{Tabla 4.}

Cumplimientos - incumplimientos.

\begin{tabular}{ccc}
\hline Respuesta & $\mathbf{f}$ & $\mathbf{p}$ \\
\hline Sí & 66 & 86.84 \\
No & 9 & 11.84 \\
No aplica & 1 & 1.32 \\
$\Sigma$ & 76 & 100.00 \\
\hline
\end{tabular}

Fuente: Lista de cotejo del cumplimiento del SGSST. Elaborado: noviembre 2019. 


\section{CIENCIAMATRIA}

Revista Interdisciplinaria de Humanidades, Educación, Ciencia y Tecnología

Año VIII. Vol. VIII. Nro 1. Edición Especial. 2022

Hecho el depósito de ley: pp201602FA4721

ISSN-L: 2542-3029; ISSN: 2610-802X

Universidad Nacional Experimental Francisco de Miranda (UNEFM). Santa Ana de Coro. Venezuela

Ángel Alberto Dionisio-Cuadrado

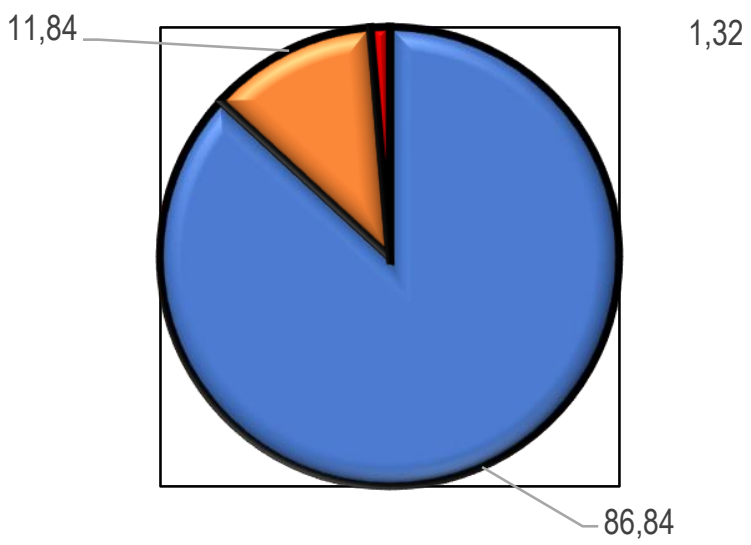

Figura 2.

Cumplimiento - incumplimiento del sistema de riesgos.

Tabla 5.

Ponderación.

\begin{tabular}{ll}
\hline Rangos de puntaje (\%) & \\
\hline Excelente & $91-100$ \\
Bueno & $81-90$ \\
Bajo & $61-80$ \\
Inaceptable & $0-60$ \\
\hline
\end{tabular}

Como se aprecia el resultado de cumplimientos está en el rango de $86.84 \%$ que significa BUENO, es decir que el Plan mejorado e implementado satisface o cumple las condiciones básicas de un sistema de gestión de riesgos, por lo que prima su elaboración e implementación, puesto que el incumplimiento es de $11,84 \%$ de acciones o 


\section{CIENCIAMATRIA}

Revista Interdisciplinaria de Humanidades, Educación, Ciencia y Tecnología

Año VIII. Vol. VIII. Nro 1. Edición Especial. 2022

Hecho el depósito de ley: pp201602FA4721

ISSN-L: 2542-3029; ISSN: 2610-802X

Universidad Nacional Experimental Francisco de Miranda (UNEFM). Santa Ana de Coro. Venezuela

Ángel Alberto Dionisio-Cuadrado

lineamientos que no se cumplen y 1 que no aplica, el alcance es bastante alto, y las garantías de previsión o evitar accidentes son bastante mejores.

¿Qué se hizo entre una y otra evaluación?, la implementación y mejora de la gestión de riesgos en cada uno de sus aspectos, como sigue:

1‥ Implementación de la política de la gestión del riesgo

$2^{\circ}$. Determinación de las responsabilidades y funciones de:

a. Coordinador del sistema de gestión de riesgo.

b. Supervisores de seguridad.

c. Los trabajadores.

d. Comité de seguridad y salud en el trabajo.

3o. Se estableció el cronograma de acciones de trabajo.

4‥ Se determinó los indicadores de gestión.

5‥ Aplicación de las encuestas de entrada - salida.

De hecho, los resultados de estos últimos en la encuesta de entrada no fueron favorables enfatizando en los resultados:

a) Sus conocimientos fueron de más del $81 \%$ entre algo hasta nada satisfactorios.

b) La sensación de inseguridad que presentaron igualmente fue del más del $81 \%$.

c) Consideraron que la situación de incidentes u ocurrencias era de alto a crítico en más de $95 \%$.

d) El $75 \%$ informaron que no han participado en la planificación de la seguridad y salud en el trabajo.

e) Más del $82 \%$ indicaron que no fueron capacitados en el tema de la seguridad y salud en el trabajo.

f) Igualmente, casi el $80 \%$ indicaron que no se cuenta con la señalética apropiada. 


\section{CIENCIAMATRIA}

Revista Interdisciplinaria de Humanidades, Educación, Ciencia y Tecnología

Año VIII. Vol. VIII. Nro 1. Edición Especial. 2022

Hecho el depósito de ley: pp201602FA4721

ISSN-L: 2542-3029; ISSN: 2610-802X

Universidad Nacional Experimental Francisco de Miranda (UNEFM). Santa Ana de Coro. Venezuela

Ángel Alberto Dionisio-Cuadrado

g) Y más del 84\% han indicado que no conocían ni tenían manejo del protocolo de gestión del riesgo.

h) Casi el $90 \%$ indicaron que no han participado, ni conocen de ello en el comité de gestión del riesgo.

i) El $75 \%$ indicaron que sus conocimientos de la gestión del riesgo no eran nada satisfactorios.

Los resultados con respecto a la encuesta de salida fueron totalmente alentadores, es necesario considerar que son referenciales, y solo obedecen a la apreciación de los participantes:

a) Sus conocimientos fueron de más del $87 \%$ entre casi a satisfactorio.

b) La sensación de seguridad que presentaron fue de más del $84 \%$.

c) Consideraron que la situación de incidentes u ocurrencias se ha reducido de $95 \%$ a $65 \%$.

d) Más del $90 \%$ informaron que les permitieron participar en la planificación de la seguridad y salud en el trabajo.

e) Más del $87 \%$ indicaron que fueron capacitados en el tema de la seguridad y salud en el trabajo luego de la implementación y mejora.

f) Igualmente, el 100\% reconocieron que la señalética era apropiada.

g) Y más del 87\% manifestaron que ya comprendían mejor el manejo del protocolo de gestión del riesgo.

h) Más del $97 \%$ indicaron que han participado y ya comprenden el rol del comité de gestión del riesgo.

i) El $87 \%$ indicaron que sus conocimientos de la gestión del riesgo eran satisfactorios. 


\section{CIENCIAMATRIA}

Revista Interdisciplinaria de Humanidades, Educación, Ciencia y Tecnología

Año VIII. Vol. VIII. Nro 1. Edición Especial. 2022

Hecho el depósito de ley: pp201602FA4721

ISSN-L: 2542-3029; ISSN: 2610-802X

Universidad Nacional Experimental Francisco de Miranda (UNEFM). Santa Ana de Coro. Venezuela

Ángel Alberto Dionisio-Cuadrado

Se presentan los datos de los accidentes e incidentes:

\section{Tabla 6.}

Evolución del reporte de incidentes.

\begin{tabular}{|c|c|c|c|c|c|c|}
\hline Respuesta & $f_{i}$ & $p_{i}$ & $f_{i}$ & $p_{i}$ & $f_{i}$ & $p_{i}$ \\
\hline Accidentes fatales & 1 & 6.67 & 0 & 0.00 & 0 & 0.00 \\
\hline Accidentes & & & & & & \\
\hline $\begin{array}{l}\text { incapacitantes } \\
\text { permanente }\end{array}$ & 3 & 20.00 & 2 & 15.38 & 0 & 0.00 \\
\hline $\begin{array}{l}\text { Accidentes } \\
\text { incapacitantes temporal }\end{array}$ & 4 & 26.67 & 4 & 30.77 & 1 & $\begin{array}{l}25.0 \\
0\end{array}$ \\
\hline Accidentes leves & 7 & 46.67 & 7 & 53.85 & 3 & $\begin{array}{l}75.0 \\
0\end{array}$ \\
\hline Sumatoria & $\begin{array}{l}1 \\
5\end{array}$ & 100.00 & 13 & 100.00 & 4 & $\begin{array}{l}100 . \\
00\end{array}$ \\
\hline
\end{tabular}

Fuente: Encuesta aplicada.

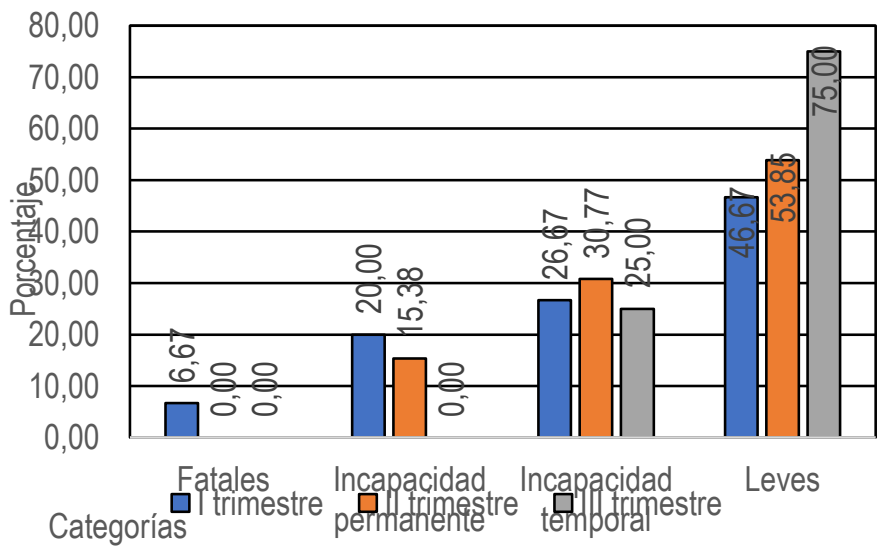

Figura 3. Registros por trimestres de los índices de accidentes. 


\section{CIENCIAMATRIA \\ Revista Interdisciplinaria de Humanidades, Educación, Ciencia y Tecnología \\ Año VIII. Vol. VIII. Nro 1. Edición Especial. 2022 \\ Hecho el depósito de ley: pp201602FA4721 \\ ISSN-L: 2542-3029; ISSN: 2610-802X \\ Universidad Nacional Experimental Francisco de Miranda (UNEFM). Santa Ana de Coro. Venezuela}

Ángel Alberto Dionisio-Cuadrado

Se puede apreciar que en el I trimestre los reportes no son nada satisfactorios, inclusive se reporta un deceso y muchos accidentes de invalidez o incapacidad permanente, en el segundo bimestre no se reportan accidentes fatales y la incapacidad permanente se reduce y es en el tercer bimestre que quedan solo dos reportes Incapacidad temporal y Accidentes leves. Se puede apreciar que los reportes de incidentes en el proceso fueron favorables, es necesario procurar que sean 0 reportes.

\section{DISCUSIÓN}

En los trabajos de investigación empleados como referentes y/o antecedentes se encuentran importantes aportes que condicen con la presente investigación y se pasan a detallar con la finalidad de realizar la comprobación de la validez de la propuesta, encontrándose relación con lo planteado por (Gonzales, 2018), por consiguiente, es necesario mejorar como se implementan las estrategias de motivación que implicó que los trabajadores sean incentivados a fomentar la Cultura de prevención y el Control de riesgos, las charlas preventivas o motivacionales ha despertado el interés por atender a salvaguardar su propia integridad en los trabajadores.

Mientras que (Atencio, 2013), considera necesario contar con un protocolo de seguridad, lo cual, contribuye a fundamentar el actual trabajo, siendo necesario asumir que se requiere una herramienta como el principal requisito a asumir e involucrar a todos en favor de ello. En contraposición, (Maylle, 2019), plantea necesario tener en cuenta en el diseño del protocolo, los riesgos: ergonómico, biológico, físico, químico y peligro emocional y los accidentes laborales existe un vínculo importante, lo que refuerza el criterio propuesto de un control estricto de riesgos contribuye a prevenir accidentes, ya que mejoran los protocolos, todo en el beneficio del estudio.

En complemento, (Arce, 2017), destaca que la atención y el establecimiento de agenda sobre la accidentalidad laboral han sido además muy poco estudiados, principalmente enfocados a pequeños periodos de muestra o a diarios locales, siendo los autores y 


\section{CIENCIAMATRIA \\ Revista Interdisciplinaria de Humanidades, Educación, Ciencia y Tecnología \\ Año VIII. Vol. VIII. Nro 1. Edición Especial. 2022 \\ Hecho el depósito de ley: pp201602FA4721 \\ ISSN-L: 2542-3029; ISSN: 2610-802X \\ Universidad Nacional Experimental Francisco de Miranda (UNEFM). Santa Ana de Coro. Venezuela}

Ángel Alberto Dionisio-Cuadrado

artículos en su mayoría provenientes de instituciones públicas. Siendo la mayor responsabilidad implementar en todos los trabajadores el mejor desempeño con seguridad en sus labores, logrando reducir significativamente los indicadores adversos en este sentido. Esto ayuda a tener referentes saludables, a diferenciar accidente de incidente, a desarrollar una mejor cultura de desempeño y/o trabajo seguro en el marco de una cultura de prevención y control de riesgos.

En cuanto a la capacitación, (Solano, 2015), indican que la falta de capacitaciones, inducciones o charlas preventivas son desfavorables y se materializan en accidentes que generan incertidumbre en el personal que labora. Mientras que (Tigre-Ortega, 2013), enfatiza el hecho de que lo importante es la necesidad de capacitar y concientizando al personal para que asuma un rol más protagónico en su propia protección y sea consciente de lo vital que es la prevención y el control del riesgo y para ello seguir los protocolos e indicaciones es fundamental.

\section{CONCLUSIONES}

Los eventos adversos que se han identificado, a partir de la información en general, se reportaron antes de la investigación: 18 de estos incidentes, entre los que sucedieron los usuales, relacionados a caídas a desnivel son los leves, 9 antes de la aplicación, 5 durante la aplicación y 3 después de la aplicación, de ellos se puede identificar los casos a desnivel, se ha elaborado una identificación en el registro y se aprecia que las caídas de desnivel siempre son reportadas como leves, por no haber sido más que incidentes en los que uno reporta una contusión leve que no requirió interrupción en las acciones de trabajo, como se aprecia en las tablas 27 a la 29 del presente estudio.

En el tiempo no se aprecian situaciones críticas o accidentes graves, como explosiones, pero si se han suscitado hechos graves antes de la aplicación del sistema se produjo un accidente mortal, ha existido y siempre existirá riesgos de explosiones, por el alto riesgo, por este tipo de empresas y sus servicios en las industrias de hidrocarburos. 


\section{CIENCIAMATRIA}

Revista Interdisciplinaria de Humanidades, Educación, Ciencia y Tecnología

Año VIII. Vol. VIII. Nro 1. Edición Especial. 2022

Hecho el depósito de ley: pp201602FA4721

ISSN-L: 2542-3029; ISSN: 2610-802X

Universidad Nacional Experimental Francisco de Miranda (UNEFM). Santa Ana de Coro. Venezuela

Ángel Alberto Dionisio-Cuadrado

Con relación a los objetivos se tiene:

a) El estudio ha podido demostrar que el sistema de gestión de riesgos influye de manera significativa en la reducción del índice de accidentabilidad, debido a que antes de la aplicación o implementación del mismo en la empresa del sub sector hidrocarburos EBH Ingenieros S.A.C. se apreciaban hasta 18 registros de accidentes e inclusive con un evento adverso fatal, después del proceso esto se ha reducido a 0 en fatales, graves y moderados y solo se reportaron 3 incidentes leves, lo que permite establecer una perspectiva de extenderlo a otras actividades en favor de la misma operatividad y logro de mejores resultados.

b) El instrumento denominado matriz de análisis del sistema de riesgos ha permitido la descripción fundamentada con normas de aspectos vitales dentro de la gestión de la seguridad y la salud en las tablas $n^{\circ} 4$ y 5 de la presente tesis, demostrando lo importante que es contar con observaciones sistemáticas y debidamente graduadas en favor de la investigación y los avances de la gestión de la seguridad y salud ocupacional.

c) Los índices de accidentabilidad se han visto reducidos de puntuaciones desfavorables en el primer trimestre al tercer trimestre, desde observar accidentes fatales, de incapacidad permanente a reducirlos y suprimirlos, logrando su control, a partir de un enfoque de cultura de prevención y control de riesgos con la herramienta sistema de gestión del riesgo, que ha demostrado su utilidad.

\section{FINANCIAMIENTO}

No Monetario. 
CIENCIAMATRIA

Revista Interdisciplinaria de Humanidades, Educación, Ciencia y Tecnología

Año VIII. Vol. VIII. Nro 1. Edición Especial. 2022

Hecho el depósito de ley: pp201602FA4721

ISSN-L: 2542-3029; ISSN: 2610-802X

Universidad Nacional Experimental Francisco de Miranda (UNEFM). Santa Ana de Coro. Venezuela

Ángel Alberto Dionisio-Cuadrado

\section{AGRADECIMIENTO}

A todos los agentes sociales involucrados en el desarrollo de la investigación.

\section{REFERENCIAS CONSULTADAS}

Aragón, C. (2016). Desarrollo de los indicadores [Development of indicators]. [Internet]. Obtenido de https://n9.cl/ohg6e

Arbaiza L., C. J. (2014). Modelo de desarrollo sostenible en la pequeña minería subterránea: Caso Kinacox [Sustainable development model in small-scale subway mining: Kinacox case study]. Lima: ESAN Ediciones. https://hdl.handle.net/20.500.12640/116

Arce G., S. E. (2017). La prevención de riesgos laborales y la accidentalidad laboral en la prensa española: representación y cobertura a partir de la Ley 31/1995 de Prevención de Riesgos Laborales (1994 - 2014) [Occupational risk prevention and occupational accidents in the Spanish press: representation and coverage from Law 31/1995 on Occupational Risk Prevention (1994 - 2014).]. Universidad de Burgos. https://dialnet.unirioja.es/servlet/tesis?codigo $=105848$

Atencio M., C. R. (2013). Estudio de accidentes laborales como acción preventiva en una empresa constructora [Study of occupational accidents as a preventive action in a construction company]. Universidad Nacional Mayor de San Marcos. https://revistasinvestigacion.unmsm.edu.pe/index.php/iigeo/article/view/11380

Diaz-Dumont, J. R., Suarez Mansilla, S. L., Nanzy, R., \& Bizarro Huaman, E. M. (2020). Accidentes laborales en el Perú: Análisis de la realidad a partir de datos estadísticos [Occupational accidents in Peru: Analysis of the reality based on statistical data]. Revista Venezolana De Gerencia, 25(89), 312-329. https://doi.org/10.37960/revista.v25i89.31533

Gonzales G., M. A. (2018). Prevención de accidentes laborales en base a un diálogo compartido en el Proyecto Ciudad Nueva Fuera Bamba [Prevention of occupational accidents based on a shared dialogue in the Ciudad Nueva Fuera Bamba Project]. Universidad Nacional de Educación. https://repositorio.unh.edu.pe/handle/UNH/1721

Hernández-Sampieri, R., Fernandez-Collado, C., \& Batista-Lucio, M. (2014). Metodología de la Investigación [Research Methodology]. McGRAW-HILL / INTERAMERICANA EDITORES, S.A. DE C.V. 
CIENCIAMATRIA

Revista Interdisciplinaria de Humanidades, Educación, Ciencia y Tecnología

Año VIII. Vol. VIII. Nro 1. Edición Especial. 2022

Hecho el depósito de ley: pp201602FA4721

ISSN-L: 2542-3029; ISSN: 2610-802X

Universidad Nacional Experimental Francisco de Miranda (UNEFM). Santa Ana de Coro. Venezuela

Ángel Alberto Dionisio-Cuadrado

Maylle A., T. C. (2019). Factores de riesgo y accidentes laborales en enfermería en un hospital público, Cercado de Lima, 2018 [Risk factors and occupational accidents in nursing in a public hospital, Cercado de Lima, 2018.]. Universidad César Vallejo. https://repositorio.ucv.edu.pe/handle/20.500.12692/31985

Miño-Cascante, G, Esparza-Paz, F, \& Esparza-Córdova, L. (2020). Niveles de accidentabilidad en operadores de Vacuums y riesgos laborales [Accident levels in

Vacuums and

risk operators]. Revista Investigación y Negocios, 13(21), 6-14.

Occupational

OIT. (2021). WHO/ILO: Nearly 2 million people die each year from work-related causes. [Internet]. retrieved from https://n9.cl/4nbsz

OMS. (2018). Caídas [Falls]. [Internet]. retrieved from https://n9.cl/gfik

PUCP - OSHAS. (2018). Medical Assistant. Obtenido de https://n9.cl/rwm77

Ramirez, E. (2004). Introducción a la Psicología [Introduction to Psychology]. Madrid: Alfaguara. Recuperado de https://n9.cl/vfwhg

Solano P., A. L. (2015). Modelo de Gestión de Seguridad y Salud Ocupacional para el control de reducción de riesgos laborales en el sector de la construcción. Cuenca. 2014 [Occupational Health and Safety Management Model for the control and reduction of occupational risks in the construction sector. Cuenca. 2014]. Universidad de Cuenca. http://dspace.ucuenca.edu.ec/handle/123456789/21978

SURA. (2020). Generalidades del sistema de riesgos laborales [General information on the labor risk system]. [Internet]. Obtenido de https://www.arlsura.com/1929

Tigre-Ortega, F. (2013). Los factores de riesgo mecánicos y su incidencia en la accidentabilidad de los trabajadores de producción de Línea Blanca de la Empresa FAIRIS C.A. [The mechanical risk factors and their incidence in the accident rate of the production workers of the White Line of FAIRIS C.A. Company.]. Universidad Técnica de Ambato. Facultad de Ingeniería en Sistemas, Electrónica e Industrial. Maestria en Seguridad e Higiene Industrial y Ambiental. http://repositorio.uta.edu.ec/handle/123456789/5736 


\section{CIENCIAMATRIA}

Revista Interdisciplinaria de Humanidades, Educación, Ciencia y Tecnología

Año VIII. Vol. VIII. Nro 1. Edición Especial. 2022

Hecho el depósito de ley: pp201602FA4721

ISSN-L: 2542-3029; ISSN: 2610-802X

Universidad Nacional Experimental Francisco de Miranda (UNEFM). Santa Ana de Coro. Venezuela

Ángel Alberto Dionisio-Cuadrado

(C2022 por los autores. Este artículo es de acceso abierto y distribuido según los términos y condiciones de la licencia Creative Commons Atribución-NoComercial-Compartirlgual 4.0 Internacional (CC BY-NC-SA 4.0) (https://creativecommons.org/licenses/by-nc-sa/4.0/). 